\title{
HELMINTOS PARÁSITOS DE Dosidicus gigas “POTA” EVISCERADA EN AREQUIPA, PERÚ
}

\section{PARASITE HELMINTHS OF JUMBO SQUID Dosidicus gigas EVISCERATE IN AREQUIPA, PERU}

\author{
Roxana Esther Céspedes ${ }^{1}$, José Iannacone ${ }^{2}$ y Alberto Salas ${ }^{3}$
}

\begin{abstract}
Resumen
El “calamar gigante” o “pota” Dosidicus gigas (D’Orbigny 1835), ha sido catalogado dentro de las especies de mayor importancia en los ecosistemas marinos pelágicos. Se cuantificó la fauna helmintológica parasitaria con énfasis en aquellos de importancia zoonótica y sanitaria de 254 individuos (D. gigas) frescos y eviscerados capturados en aguas oceánicas del Pacifico Sur de

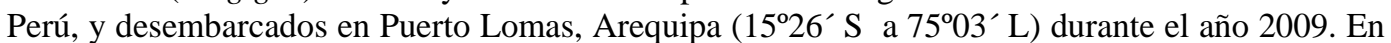
cada pota se determinó su Longitud Dorsal del Manto (LDM) en cm y su Peso Estándar del Manto (PEM) en kg. Se encontraron cinco taxones larvales de helmintos: Didimozoidea sp. no identificada (Abundancia media de infección (AM):0,0039; prevalencia:0,39\%), dos céstodos: Hepatoxylon trichiuri (Holten 1802) (AM:0,04; prevalencia:0,78\%) y Tentacularia coryphaenae (Bosc 1797) (AM:1,68; prevalencia:56,29\%), y dos nemátodos Anisakis simplex o Tipo I (Dujardin 1845) (AM:0,23; prevalencia:15,74\%), A. physeteris o Tipo II (Baylis 1923) (AM:1,11; prevalencia: 50\%). Se encontró el 57\% de calamares (D. gigas) infestados por el nemátodo zoonótico Anisakis. Los tres parásitos con mayor importancia específica fueron: T. coryphaenae, A. physeteris y A. simplex. Se encontró una relación lineal con la LDM del hospedero y la prevalencia media de T. coryphaenae. Se observó una relación lineal con el PEM y la prevalencia media de A. simplex. Los estimadores no paramétricos indicaron que se requiere un incremento en el esfuerzo de colecta y señalaron que deberían encontrarse hasta seis especie helmínticas en $D$. gigas. La comparación de la LDM entre las potas parasitadas y no parasitadas por A. simplex, A. physeteris y T. coryphaenae no mostró diferencias significativas. Finalmente, se realizó un análisis comparativo de los parásitos de D. gigas entre los años 1981-1989, 2003-2004, 2008-2009 y 2009.
\end{abstract}

Palabras clave: Anisakis, Hepatoxylon, Tentacularia.

\begin{abstract}
The giant squid or squid Dosidicus gigas (D'Orbigny 1835) has been included in the list of most important species in pelagic marine ecosystems. Parasitic helminthes fauna with emphasis on those of zoonotic and sanitary importance was assessed from 254 fresh and eviscerated individuals (D. gigas) caught in oceanic waters of the Pacific Ocean, south of Peru, and landed in Puerto Lomas, Arequipa ( $15^{\circ} 26$ 'S to $\left.75^{\circ} 03^{\prime} \mathrm{L}\right)$ during the year 2009. Dorsal Length (MDL) in cm. and Weight of Standard Mantle (MSW) in kg. was determined in each squid. Five different helminthes larval taxa were found: Didimozoidea unidentified sp. (mean abundance of infection (MA): 0.0039; Prevalence: 0.39\%), two larval cestodes: Hepatoxylon trichiuri (Holten 1802) (MA: 0.04, prevalence: 0.78\%) and Tentacularia coryphaenae (Bosc 1797) (MA: 1.68, Prevalence: 56.29\%), and two nematodes Anisakis simplex or type I (Dujardin 1845) (MA: 0.23; Prevalence: 15.74\%), A. physeteris or Type II (Baylis 1923) (MA: 1.11, Prevalence: 50\%). 57\% of squid ( $D$. gigas) were infested by the zoonotic nematode Anisakis. The three parasites with higher specific importance were: T. coryphaenae, A. physeteris and A. simplex. A linear relationship with the MDL of the host and the average prevalence of $T$. coryphaenae was found. A linear relationship with the MSW and the mean prevalence of A. simplex was found. Nonparametric estimators indicated that an increase in the collection effort was needed and indicated that there should be up to six helminth species in D. gigas. Comparison of MDL between parasitized and non-parasitized squids by $A$. simplex, $A$. physeteris, $T$. coryphaenae showed no significant differences. Finally, a comparative quantitative analysis of the components of the parasitic fauna of D. gigas between years 1981-1989, 2003-2004, 2008-2009 and 2009 was performed.
\end{abstract}

Key words: Anisakis, Hepatoxylon, Tentacularia. 


\section{Introducción.}

Dosidicus gigas (D’Orbigny 1835) conocido vernacularmente como "pota" o "calamar gigante" es uno de los cefalópodos de mayor tamaño y uno de los calamares más abundantes en los ambientes pelágicos (Roper et al., 1984). Esta especie es endémica del Pacífico sureste y se distribuye entre los $37-40^{\circ} \mathrm{N}$ y $45-47^{\circ} \mathrm{S}$ llegando incluso a los $52^{\circ} \mathrm{S}$ y entre los 125 y $140^{\circ} \mathrm{W}$ (Nesis,1970a,b; Benites \& Valdivieso, 1986).

Han sido registrados los siguientes helmintos en “pota” D. gigas: Didymozoidae sp. no indentificada, Pelichnibothrium speciosum (Monticelli 1889), Phyllobothrium sp., Tentacularia coryphaenae (Bosc 1797), Hepatoxylum trichuri (Holten,1802), Anisakis simplex o tipo I (Dujardin, 1845), Anisakis physeteris o tipo II (Baylis 1923), Porrocaecum sp., Contracaecum sp. y Spinitectus sp. (Gaevskaya et al., 1982, 1983, 1987; Gaevskaya \& Shukhgalter, 1992; Shukhgalter, 1985, 1988a,b. 1992; Shukhgalter \& Nigmatullin, 2001; Iannacone \& Alvariño, 2009; Pardo-Gandarillas et al., 2009).

En estudios realizados en el Pacífico Central, se ha registrado la presencia de la post larva de $T$. coryphaenae en los órganos internos de la cavidad del manto y algunas de ellas dentro del manto de la pota. Metacercarias de didymozoidea (Tremátoda) han sido observadas en la pared interna del estómago, y ocasionalmente en el esófago, y en el ciego, a lo largo de los principales vasos sanguíneos, y a veces libre en la cavidad del estómago (Shukhgalter \& Nigmatullin, 2001). Estas larvas de didymozoideos han sido encontrados en $D$. gigas en el Golfo de California (Hochberg, 1990; Overstreet \& Hochberg, 1975) y al sur este del Pacífico (Gaevskaya et al., 1982, 1983, 1987; Gaevskaya \& Shukhgalter, 1992; Shukhgalter, 1986, 1988b).

En el cefalópodo D. gigas, A. simplex y A. physeteris se localizan dentro del tracto digestivo, en la cavidad peritoneal o en las vísceras en forma libre e inclusive enquistadas en la musculatura en forma espiral. Cuando los cefalópodos son ingeridos por cetáceos y pinnípedos, las larvas penetran en la mucosa gástrica donde sufren las dos últimas mudas y alcanzan la madurez sexual, cerrando así el ciclo vital del parásito. La anisakiosis es una parasitosis humana zoonótica del tracto digestivo (Iannacone \& Alvariño, 2009).

La anisakidosis por larvas de $A$. physeteris probablemente emergió durante el fenómeno de El Niño ocurrido en la costa peruana en los años 199798, el que se explicaría por los siguientes factores: en primer lugar, por el volumen de desembarque de Coryphaena hippurus (Linneo 1758) ("perico" o "pez delfín”) producto de la pesca artesanal entre enero y marzo de 1998. En segundo lugar, por el posible incremento del consumo del "perico" bajo la forma de (cebiche) por la población debido a la notable disminución de la captura de otras especies propias de aguas frías, este hecho pudo incrementar la probabilidad del riesgo de infección. En tercer lugar, a la prevalencia e intensidad media elevada de larvas de A. physeteris en el "perico" durante El Niño (1997-98) (Cabrera \& Trillo, 2004).

El control de calidad y la inspección sanitaria de la "pota" $D$. gigas es importante debido a que los parásitos de interés zoonótico como los ascaroideos de la familia Anisakidae son transmitidos al hombre cuando se ingiere este alimento crudo o sin cocción. De igual forma las larvas de los céstodos $T$. coryphaenae $y \quad H$. trichiuri aunque no son considerados helmintos zoonóticos ocasionan por su gran tamaño un efecto estético negativo y por ende un rechazo de este producto alimenticio por el consumidor (De la Torre et al., 2000; Cabrera \& Suárez-Ognio, 2002).

Los estudios comunitarios de los parásitos de organismos marinos en el Pacífico Sur es una línea de investigación acrecentada en los últimos años (Iannacone et al., 2003, 2008; Iannacone \& Alvariño, 2009).

El objetivo de la presente investigación fue determinar la comunidad de helmintos en $D$. gigas con énfasis en aquellos de importancia zoonótica como los anisákidos en la zona Sur del Perú.

\section{Materiales y métodos. \\ Dosidicus gigas}

El material biológico para el presente estudio fue obtenido de 254 muestras de D. gigas "pota" frescas y evisceradas (con ausencia de vísceras) en 2 períodos, durante los días 7, 21, 22 y 23 de febrero y 7 y 14 de Marzo del 2009 del Puerto Lomas (Arequipa), Perú $\left(15^{\circ} 26^{\prime} \mathrm{LS} ; 7^{\circ} 03^{\prime} \mathrm{LW}\right)$ provenientes de la zona de pesca 18 millas rumbo 350 Asia 6. En todos los especímenes "potas" se determinó: la Longitud Dorsal del Manto en cm (LDM) mediante una guincha y el Peso Estándar del Manto en Kg (PEM). Cada ejemplar se colocó ventralmente sobre la mesa de disección del Puerto de Lomas en Arequipa para tomar la medida. El manto fue medido desde el extremo anterior hasta el extremo posterior dorsalmente. También se pesó el manto en una balanza electrónica (Marca: Mitzuco ${ }^{\circledR}$ ) de $500 \mathrm{~kg}$.

Helmintos

Para la extracción de los helmintos se colocó el manto de cada individuo "pota" dorsalmente sobre la mesa, y luego mediante cortes con ayuda de un bisturí y de una tijera quirúrgica se procedió a realizar cortes longitudinales en la musculatura del manto en la mesa de disección del Puerto Lomas en Arequipa.

El protocolo utilizado para la obtención de los parásitos fue el de Zuev et al. (1985). Los métodos utilizados siguieron el enfoque estándar empleado para todos los helmintos que se identificaron en las "potas" frescas. Las larvas de helmintos fueron extraídas con ayuda de un bisturí, estilete, pinzas 
delgadas y luego fueron observadas con ayuda de un Estereoscopio (Olympus América Latina ${ }^{\circledR}$ ) y se procedió a la determinación de los helmintos endoparásitos. Los tremátodos y céstodos fueron fijados y almacenados en alcohol al 70\% en frascos de vidrio "viales". Para la fijación y preservación de nemátodos se siguió a Eiras et al. (2000). Después de la colección de los nemátodos, se lavaron en solución salina $0,85 \%$. Aparte, en un tubo de ensayo, se calentó alcohol de $70 \%$ hasta $70^{\circ} \mathrm{C}$. Se retiró la mayor cantidad de solución posible en el que se encontraban los especimenes y se agregó rápidamente el alcohol caliente. De este modo los nemátodes murieron estirados. Los nematodos que se encontraron muertos, fueron incorporados directamente en alcohol al 70\%. Después de estudiarlos, los nemátodes se guardaron en alcohol de $70 \%$ en viales de vidrio de $5 \mathrm{~cm}$ de altura con tapa de goma. Todas las muestras incluyeron una etiqueta $2 \mathrm{~cm} \times 3 \mathrm{~cm}$ de papel canson en la que se colocó los siguientes datos: 1) Nombre y localización del parásito; 2) Nombre científico y vulgar del huésped; 3) Procedencia del huésped; 4) Fecha de colecta; 5) Nombre del colector y 6) Número de registro (el mismo de la libreta o ficha de recolección). La fijación y rotulación de los helmintos se realizó en el Laboratorio de Microbiología del Instituto Tecnológico Pesquero del Perú (ITP). El material representativo fue depositado en el Museo de Historia Natural (MHN) de la Universidad Ricardo Palma (URP).

La identificación de los helmintos se realizó en el Laboratorio de Microbiología del Instituto Tecnológico Pesquero del Perú (ITP), Carretera a Ventanilla Km. 5,2, Callao y en el Laboratorio de Parasitología (Laboratorio de Invertebrados 201) de la Facultad de Ciencias Biológicas de la Universidad Ricardo Palma, Santiago de Surco, Lima, Perú. Para la determinación de las larvas de céstoda se hizo de acuerdo a Joyeux \& Baer (1936), Dollfus (1942), Yamaguti (1959), Stunkard (1977), Gaevskaya (1977), Palm (1995), Scholz et al. (1998), Palm \& Walter (2000) y Palm (2004). Las larvas del nemátodo Anisakis de acuerdo con Berland (1961), Koyama et al. (1969), Smith \& Wootten (1978), Smith (1983) y Matsuura et al. (1992).

Análisis de datos

Se determinó en los hospederos la LDM (Longitud Dorsal del Manto), la cual se dividió en cinco rangos de 6,6 cm cada uno aplicando la regla de Sturges. Los PEM (Peso Estándar del Manto) en los hospederos se dividieron en ocho rangos de aproximadamente 0,76 Kg y también se aplicó la regla de Sturges.

La terminología de los descriptores ecológicos (prevalencia, abundancia e intensidad media de infección) siguió los criterios de Bush et al. (1997). Se determinaron los descriptores parasitológicos: prevalencia, abundancia e intensidad media para todos los parásitos encontrados. La prevalencia de infección se calculó como la relación entre el número de hospederos parasitados de una especie particular de parásito dividido entre el número total de hospederos examinados y expresado en porcentaje. La abundancia media de infección fue calculada como el número de individuos de una especie particular de parásito en una muestra de una especie hospedera dividido entre el número total de hospederos examinados (incluye los hospederos infectados y no infectados). La intensidad media de infección se determinó como el número total de parásitos de una especie particular encontrados en una muestra dividido entre el número de hospederos infectados con el parásito. La prevalencia y la abundancia total se calcularon en base al número total de potas parasitadas y del total de parásitos para todas las especies encontradas, independiente de su identidad taxonómica, respectivamente. El Índice de dispersión (ID) empleado, se determinó de la relación entre Varianza $\left(S^{2}\right)$ /abundancia media con el fin de indicar si la especie presenta una distribución contagiosa, uniforme o al azar. El análisis de los parásitos se hizo solo para las especies con prevalencias mayores al $5 \%$, de acuerdo a Esch et al. (1990). Con el fin de obtener un índice integrado entre la prevalencia y abundancia se empleó el concepto de importancia específica (I) estimado como la influencia de las especies parásitas en la comunidad fue calculado: $\mathrm{I}=$ Prevalencia relativa + (abundancia relativa) x 100 (Bursey et al., 2001).

Los datos de prevalencia para los helmintos principales (> 45\%), secundarios (entre 10 y 45\%) y raros $(<10 \%)$ se calcularon para toda la muestra evaluada. La frecuencia de dominancia y la dominancia relativa (número de especimenes de una especie / número total de especimenes de todas las especies en la infracomunidad) de cada especie parásita fue calculada de acuerdo a Alves et al. (2002). El coeficiente de correlación de Pearson fue empleado para evaluar la asociación entre la LDM y PEM de $D$. gigas con la prevalencia de infección, previa transformación angular de los valores de prevalencia. Se realizó una comparación entre las potas parasitadas y no parasitadas con relación a la LDM y se evaluó utilizando la prueba de $\mathrm{t}$ de Student, previa transformación de los datos a logaritmo.

Los índices estimadores del esfuerzo de muestreo de los parásitos de $D$. gigas son un conjunto de estimadores no-paramétricos en el sentido estadístico, ya que no asumen el tipo de distribución del conjunto de datos y no los ajustan a un modelo determinado y requieren solamente datos de presencia-ausencia y fueron empleado para verificar si el esfuerzo del muestreo fue óptimo de acuerdo a la cantidad de especies encontradas (Moreno, 2001). Se emplearon tres métodos no paramétricos: 
Chao-2, Jacknife de primer orden, Jacknife de segundo orden para estimar el número máximo de riqueza de parásitos a partir del esfuerzo de muestreo realizado y de la acumulación de nuevos taxa que van apareciendo en las potas muestreadas durante el 2009 (Moreno, 2001).

Los índices de Sörensen cualitativo y cuantitativo fueron empleados para comparar la abundancia de la parasitofauna de $D$. gigas entre 1981-1989 (Shukhgalter \& Nigmatullin, 2001), 2003-2004 (Pardo-Gandarillas et al., 2009) y el 2008-2009 (Iannacone \& Alvariño, 2009). Se realizo un análisis comparativo cuantitativo de los componentes de la fauna parasitaria de D. gigas entre los cuatro periodos; la variación entre años en el número de potas, LDM promedio, rango de la LDM y las características de la diversidad parasitaria alfa y beta de la pota entre los años 1981-1989, 20032004, 2008-2009 y 2009 (presente estudio).

La diversidad parasitaria alfa fue calculada a través del índice de Shannon Weaver $\left(\mathrm{H}^{\prime}\right)$, de Equitabilidad (J), de dominancia numérica de Berger-Parker (d), y de dominancia de Simpson (S) para 1981-1989, 2003-2004, 2008-2009 y 2009 ((Moreno, 2001; Iannacone et al., 2003). No se incluyó a $P$. speciosum en los cálculos de la diversidad parasitaria alfa debido a que durante el periodo 1981-1989 se colectaron 564160 especímenes, y en adición durante el periodo 20032004 no es indicada la cantidad de especimenes colectados de dicha especie (Shukhgalter \& Nigmatullin, 2001; Pardo-Gandarillas et al., 2009). El nivel de significancia fue evaluado a un alfa $=0,05$. Para el cálculo de las pruebas estadísticas descriptivas e inferenciales se usó el paquete estadístico SPSS 15,0 .

\section{Resultados.}

Del estudio realizado en el presente trabajo se determinó la fauna helmintológica a 254 individuos de "pota” frescas y evisceradas. Con relación a la catalogación en relación a la importancia de cada especie empleando los datos de prevalencia: $T$. coryphaenae fue considerada principal; A. physeteris y A. simplex fue secundaria; y finalmente dos helmintos larvarios fueron considerados raros, Didimozoidea sp. no identificada (Tremátoda) y de $H$. trichiuri (Tabla 1). La Tabla 1 muestra la prevalencia, intensidad y abundancia media de infección de los cinco endoparásitos encontrados en los 254 hospederos muestreados de D. gigas. Los tres parásitos con mayor importancia específica fueron $T$. coryphaenae, A.

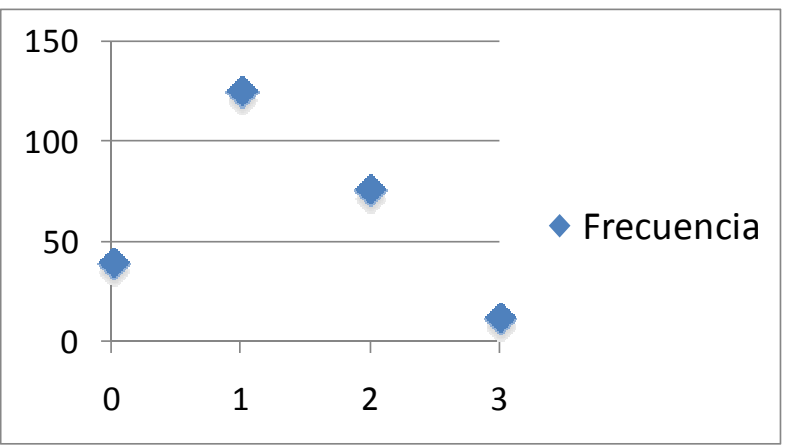

Figura 1. Frecuencia de riqueza de especies parasitas de D. gigas (Cephalopoda) del Puerto Lomas, Arequipa, Perú.

physeteris y A. simplex. La Figura 1 nos indica el siguiente orden decreciente: $1>2>3$ especies de helmintos por pota.

En tres parásitos (T. coryphaenae, A. physeteris y A. simplex) se observó una distribución sobredispersa (distribución binomial negativa), pues el índice de dispersión fue mayor a 1 (Figura 2), lo cual indica que pocas potas presentaron la mayor carga parasitaria de estos tres helmintos. Para el caso de Didymozoidae y de $H$. trichiuri por presentar prevalencias menores al 5\% no se determinó su índice de dispersión.

Las mayores frecuencias de dominancia $\mathrm{y}$ dominancia relativa fueron para $T$. coryphaenae y $A$. physeteris (Tabla 2). La Tabla 3 indica una relación lineal con la LDM del hospedero y la prevalencia media de $T$. coryphaenae. La prevalencia de $A$. physeteris y A. simplex y del parasitismo global no presentaron una relación lineal con la talla del hospedero.

La Tabla 4 indica la relación lineal con el Peso Estándar del Manto (PEM) (kg) del hospedero y la prevalencia media de $A$. simplex. La prevalencia de $A$. physeteris, de $T$. coryphaenae y el parasitismo global no presentaron una relación lineal con el peso del manto del hospedero (Tabla 4). La Tabla 5 indica que 
no existe relación entre la longitud dorsal del manto (LDM) y peso estándar del manto (PEM) de D. gigas con relación a la abundancia media de infección.

Tabla 2.- Frecuencia de dominancia y frecuencia de dominancia compartida de los helmintos parásitos de Dosidicus gigas en el Puerto Lomas, Arequipa, Perú.

\begin{tabular}{ccc}
\hline Parásitos & $\begin{array}{c}\text { Frecuencia } \\
\text { de } \\
\text { dominancia }\end{array}$ & $\begin{array}{c}\text { Frecuencia de } \\
\text { dominancia } \\
\text { compartida } \\
\text { de dos o más } \\
\text { especies }\end{array}$ \\
\hline $\begin{array}{c}\text { Didimozoidea sp. } \\
\text { no identificado }\end{array}$ & 0 & 1 \\
Hepatoxylon trichiuri & 2 & 0 \\
Tentacularia coryphaenae & 104 & 14 \\
Anisakis simplex & 9 & 7 \\
Anisakis physeteris & 78 & 18 \\
\hline
\end{tabular}

Se realizó un análisis comparativo cuantitativo de los componentes de la fauna parasitaria de $D$. gigas entre los años 1981-1989, 2003-2004, 2008-2009 y 2009 (presente estudio) en donde los resultados muestran que la prevalencia de la especie A. simplex o tipo I y A. physeteris o tipo II es mayor en el año 2009 $\mathrm{y}$ menor en el año 2003-2004 y 2008-2009 respectivamente. Didymozoidae es mayor en los años 1981-1989 y está ausente en los años 2003-2004; la especie $T$. coryphaenae registra una mayor prevalencia en los años 2008-2009; la especie $H$. trichiuri fue mayor en el año 2003-2004. En cuanto a la abundancia media las especie A. simplex o tipo I presentó el mismo valor en los años 1981-1989 y 2009, y la especie A. physeteris o tipo II es mayor en el año 1981-1989; el taxa Didymozoidae gen. sp. no identificada fue mayor en los años 1981-1989 y está ausente en los años 2003-2004; T. coryphaenae registrá una mayor abundancia media en los años 2008-2009. Finalmente, $H$. trichiuri fue mayor en el año 2003-2004 (Tabla 7).

En la Tabla 8 se señala una variación entre años en donde el mayor número de potas colectadas fue en

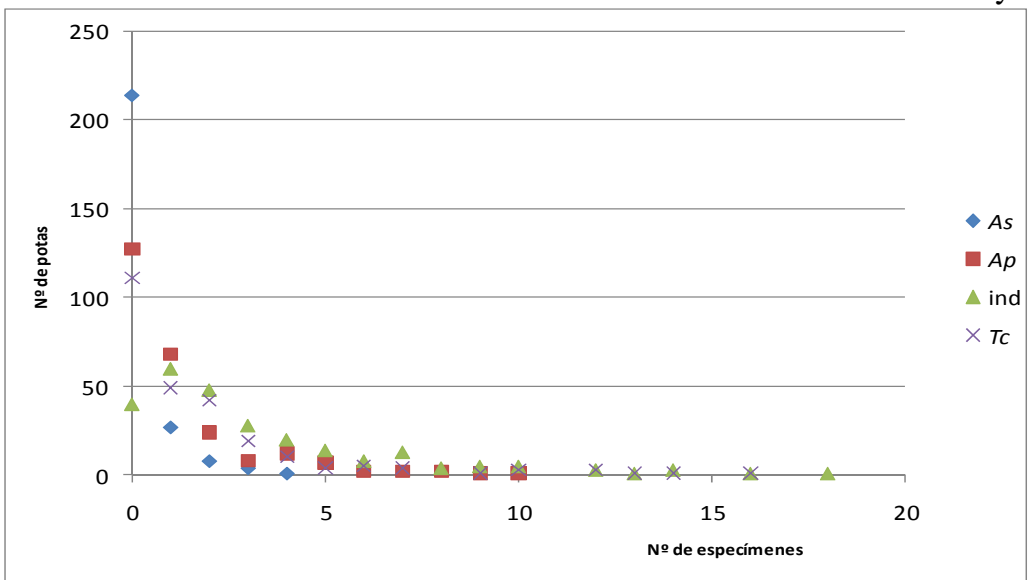

Figura 2. Frecuencia del número de parásitos encontrados en Dosidicus gigas. As = Anisakis simplex. Ap = Anisakis physeteris. $\mathrm{Tc}=$ Tentacularia coryphaenae. Ind $=$ Individuos o especimenes totales.

En relación a las abundancias monoespecíficas y recurrentes se constató que de acuerdo a los valores obtenidos las especies $A$. simplex y $A$. physeteris tienen una disminución de la abundancia media cuando ambas especies se encuentran juntas (Tabla 6)

Los tres estimadores no-paramétricos Chao-2, Jacknife-1 y Jacknife-2 en forma coincidente señalaron un valor 6 en relación al número de especies parásitas que debieron encontrarse en la muestra. La acumulación del número de especies encontradas en los hospederos se mantiene constante a un valor de 5, partir del espécimen 211 y luego no existe una mayor aparición de especies nuevas en los siguientes hospederos muestreados (Figura 3).
1981 y 1989; la mayor LDM promedio fue en 2008-2009, la mayor riqueza de especies encontradas fue en 1981-1989. En cuanto a los Índices de diversidad alfa se deduce que existió aumento de especies y mayor equidad de especies en los años 1981-1989. Hubo una mayor similaridad cualitativa de la fauna parasitaria de la pota entre los años 2003-2004 versus 2009 (80\%) y una mayor similaridad cuantitativa en los años 1981-1989 y 2009 (26,23\%).

La comparación entre la LDM (cm) entre las potas parasitadas y no parasitadas con A. simplex, A. physeteris, T. coryphaenae y parásitos totales fue similar. (Tabla 9).

\section{Discusión.}

Todos los endoparásitos de la pota de la zona Sur del Perú, se encontraron en estado larval, por lo que la pota sería el hospedero intermediario o paraténico, según el caso, para las cinco especies de helmintos, los cuales alcanzan su madurez sexual en peces óseos o cartilaginosos, mamíferos o aves marinas. Los cestodos fueron encontrados en estado de larva plerocercoide para $T$. coryphaenae lo que implica que la pota es el hospedero intermediario para este parásito y los posibles definitivos son Prionace glauca Linnaeus 1758 y Carcharinus spp. Los nemátodos anisákidos en estadio larvario $\left(\mathrm{L}_{3}\right)$ : $A$. simplex y $A$. physeteris presentan a la pota como hospedero de 
Tabla 3.- Intervalos (I), Medias (M), Frecuencias (F) de la Longitud Dorsal del manto (cm) de Dosidicus gigas eviscerada versus la Prevalencia (PREV) de los endoparásitos en el Puerto Lomas, Arequipa, Perú.

\begin{tabular}{ccccccc}
\hline \multirow{2}{*}{$\begin{array}{c}\text { I de LDM } \\
(\mathrm{cm})\end{array}$} & $\begin{array}{c}\text { M de LDM } \\
(\mathrm{cm})\end{array}$ & $\mathrm{F}$ & & \multicolumn{5}{c}{ PREV } \\
\cline { 4 - 7 } & & & & Anisakis simplex & $\begin{array}{c}\text { Anisakis } \\
\text { physeteris }\end{array}$ & $\begin{array}{c}\text { Tentacularia coryphaenae } \\
\text { Parasitismo } \\
\text { global }\end{array}$ \\
\hline$<44,07$ & 36,71 & 4 & 0 & 75 & 25 & 75 \\
$44,08-51,43$ & 47,75 & 25 & 20 & 64 & 60 & 96 \\
$51,44-58,79$ & 55,11 & 60 & 11,66 & 48,33 & 45 & 81,66 \\
$58,80-66,15$ & 62,47 & 127 & 18,11 & 44,01 & 54,33 & 83,46 \\
$66,16-73,51$ & 69,83 & 32 & 9,37 & 50 & 71,87 & 87,75 \\
$>73,51$ & 80,87 & 6 & 33,33 & 50 & 83,33 & 100 \\
$r$ & & & 0,72 & $-0,77$ & 0,89 & 0,65 \\
P & & & 0,10 & 0,07 & 0,01 & 0,17 \\
\hline
\end{tabular}

$\mathrm{r}=$ coeficiente de correlación de Pearson. $\mathrm{P}$ = significancia.

transporte. Anisakis simplex o tipo I presenta como hospedero definitivo a Balaenoptera spp. y a Tursiops truncatus (Montagu 1821) y A. physeteris o Tipo II a

Physeter macrocephalus (Linnaeus 1758) (Iannacone \& Alvariño, 2009).

El ciclo de los didymozoideos gen sp. no identificada es complejo, y se acepta que requiere de tres a cuatro hospederos para completarlo (Gómez et al., 2007). Larvas metacercarias de trematodos de la familia didimozoidae han sido registrados a nivel de su cavidad celómica en animales planctónicos como los chaetognatos y crustáceos, quienes se comportan como segundos hospederos intermediarios. Los cirripedos adultos del género Lepas Linnaeus 1758 han sido involucrados como segundos hospederos intermediarios (Gómez et al., 1999, 2007). En el caso de los peces de la familia Lethrinidae y Serranidae son considerados terceros hospederos intermediarios. Los cefalópodos a nivel del tracto digestivo y muchos peces están involucrados como hospederos paraténicos. La metacercaria de los didymozoideos no

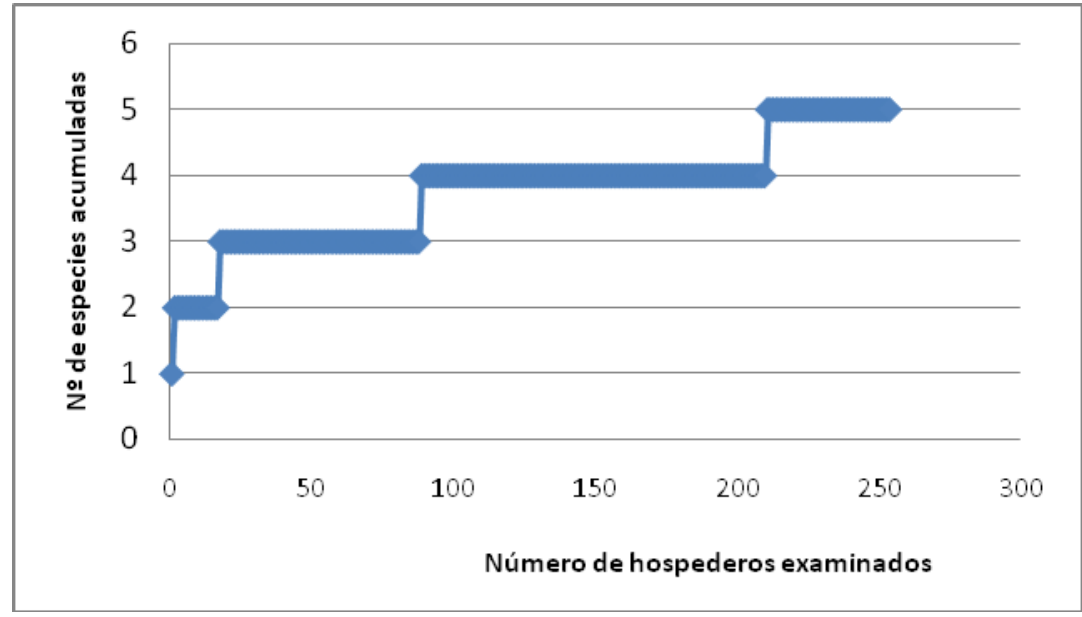

Figura 3. Curva de frecuencia acumulada de especies parásitas en relación al número de Dosidicus gigas (Cephalopoda) muestreadas. muestra ninguna preferencia por ninguno de los hospederos paraténicos y se puede encontrar en peces cartilaginosos como Rhinobatidae y en peces óseos como Clupeiformes, Atheriniformes, Beloniformes,

Scorpaeniformes, Perciformes, Pleuronectiformes y Tetraodontiformes (Rodriguez et al., 2004; Gómez et al., 2007). Finalmente los peces marinos pelágicos de gran tamaño de la familia Scombridae, sub familia Thunninae son los hospederos finales o cuartos hospederos (Justo \& Kohn, 2009). Las larvas de didimozoideos en $D$. gigas se han encontrado en el golfo de California y en el Pacífico Sur-Este (Shukhgalter \& Nigmatullin, 2001). En el presente trabajo se han observado una metacercaria de didimozoidea en restos de la pared interna del estómago en potas evisceradas de $64 \mathrm{~cm}$. Nigmatullin et al. (2001) indica que las potas de menor tamaño de 30 a $360 \mathrm{~mm}$ se alimentan de invertebrados planctónicos y de larvas de peces que servirían de hospederos intermediarios y paraténicos de didimozoideos. Las potas de mayor tamaño por el tipo de dieta no presentaría esta clase de larvas metacercarias. Por lo que al parecer el hallazgo de esta larva metacercaria en la pota de este tamaño sería un hecho inusual.

Hepatoxylon trichiuri ha sido aislada en diversas condiciones ambientales y áreas geográficas cosmopolitas, principalmente en peces de profundidad (Maladineo, 2006; Pardo-Gandarillas et al., 2009). Las 
Tabla 4.- Intervalos (I), Medias (M), Frecuencias (F) de los Pesos estándares (Kg) de Dosidicus gigas eviscerada versus la Prevalencia (PREV) de los endoparásitos en el Puerto Lomas, Arequipa, Perú. Intervalos (I), Medias (M), Frecuencias (F) y Prevalencia (PREV) de de Dosidicus gigas infectados de dos parásitos en el Puerto Lomas, Arequipa, Perú.

\begin{tabular}{ccccccc}
\hline \multirow{2}{*}{$\begin{array}{c}\text { I de PEM } \\
(\mathrm{Kg})\end{array}$} & $\begin{array}{c}\mathrm{M} \\
\mathrm{de}\end{array}$ & $\mathrm{F}$ & \multicolumn{5}{c}{ PREV } \\
\cline { 5 - 7 } & PEM $(\mathrm{Kg})$ & & Anisakis simplex & Anisakis physeteris & Tentacularia coryphaenae & Total \\
\hline $1,50-2,26$ & 1,88 & 7 & 14,28 & 42,85 & 85,71 & 100 \\
$2,27-3,03$ & 2,65 & 40 & 10 & 50 & 55 & 85 \\
$3,04-3,80$ & 3,42 & 66 & 12,12 & 54,54 & 46,96 & 83,33 \\
$3,81-4,57$ & 4,19 & 85 & 18,82 & 42,35 & 54,11 & 84,70 \\
$4,58-5,34$ & 4,96 & 25 & 16 & 48 & 60 & 84 \\
$5,35-6,11$ & 5,73 & 15 & 20 & 66,66 & 60 & 73,33 \\
$6,12-6,88$ & 6,50 & 12 & 25 & 75 & 75 & 91,66 \\
$>6,89$ & 7,46 & 4 & 25 & 25 & 75 & 75 \\
r & & & 0,89 & 0,05 & 0,16 & $-0,59$ \\
P & & & 0,003 & 0,89 & 0,69 & 0,12 \\
\hline
\end{tabular}

$\mathrm{r}=$ coeficiente de correlación de Pearson. $\mathrm{P}$ = significancia.

Tabla 5.- Valores de los coeficientes de correlación de Pearson usados para evaluar la relación posible entre la longitud dorsal de manto (LDM) y peso estándar del manto de Dosidicus gigas (PEM) y la abundancia de sus parásitos componentes de su comunidad parasitaria y del parasitismo global en el Sur del Perú. (p) nivel de significancia, (rs) coeficiente de correlación. (*) Longitud dorsal del manto vs abundancia media $(* *)$ pesos estándares vs abundancia media.

\begin{tabular}{lcccc}
\hline \multicolumn{1}{c}{ Parásito } & $\mathrm{r}^{*}$ & $\mathrm{p}$ & $\mathrm{r}^{* *}$ & $\mathrm{P}$ \\
\hline Tentacularia coryphaenae & 0,11 & 0,06 & 0,07 & 0,24 \\
Anisakis simplex & 0,043 & 0,49 & 0,09 & 0,14 \\
Anisakis physeteris & 0,020 & 0,75 & 0,07 & 0,21 \\
Parasitismo global & 0,11 & 0,06 & 0,11 & 0,06 \\
\hline
\end{tabular}

formas larvarias plerocercoides o post larvas sin blastocistos de $H$. trichiuri han sido empleadas como un indicadores biológicos de stock poblacionales a nivel muscular o mesenterios en peces principalmente de la familia Scombridae y Merluccidae, y en muchos otros teleósteos, que se comportan como intermediarios o paraténicos (Dollfus, 1942; Campbell \& Callahan, 1998; Maladineo, 2006). Las formas adultas o post larvales de este céstodo trypanorhynchido parasitan a peces cartilaginosos pelágicos como la “tintorera” P. glauca (Palm, 1995; Campbell \& Callahan, 1998; Knoff et al., 2004; Palm, 2004). En Puerto Lomas, Arequipa, Perú se le ha en encontrado en $D$. gigas con una prevalencia muy baja (Tabla 1). Sin embargo, es la forma larvaria de helminto de mayor intensidad media de infección en D. gigas en el presente estudio. Iannacone \& Alvariño (2009) para la zona norte del Perú en potas mayores a $53,5 \mathrm{~cm}$ no encuentran este helminto parásito. PardoGandarillas et al. (2009) registran a larvas plerocercoides de este helminto con prevalencias sobre el $70 \%$ en $D$. gigas del Sur-Centro de Chile, y señalan es muy común en diversos peces hospederos en Chile. Este último autor argumenta que las placas de Nazca actúan en el Perú como una barrera para la migración del stock poblacional de $D$. gigas de Chile al Perú. Por ende, al encontrarse Punta Lomas en Arequipa previa a las placas de Nazca en el Perú en dirección Chile-Perú, aun ha registrado a $H$. trichiuri en $D$. gigas pero con una baja prevalencia.

Nigmatullin et al. (2009) en el cefalópodo Ommastrephes bartramii (Lesueur 1821) ha registrado al helminto $T$. coryphaenae muy móvil y capaz de migrar en la cavidad del manto desde las gónadas (Pardo-Gandarillas et al., 2009). En adición existiría una estrecha relación entre $D$. gigas alimento clave de tiburones como P. glauca, depredador generalista y oportunista, y la transmisión de $T$. coryphaenae desde D. gigas a este pez cartilaginoso marino (PardoGandarillas et al., 2007). Al aumentar la longitud de los tiburones, éstos podrían consumir potas de mayor tamaño y por ende facilitar la sincronización del ciclo biológico de $T$. coryphaenae con potas como hospederos paraténico y P. glauca como hospedero definitivo. Este taxa se ha registrado en otras 20 especies de moluscos cefalópodos. El aumento en la dieta de crustáceos planctónicos que portarían las larvas procercoides de $T$. coryphaenae como primeros hospederos intermediarios y principalmente de peces paraténicos que en el mar del Perú serian: C. hippurus, Merluccius gayi peruanus (Ginsburg 1954), Scomber japonicus peruanus Houttuyun 1782 y Trachurus pincturatus murphyi Nichols 1920 (Iannacone \& Alvariño, 2009), transportarían las larvas plerocercoides que permitirían una acumulación en el tiempo y un aumento de la prevalencia de este helminto con la talla de $D$. gigas. 

especies de Anisakis en Dosidicus gigas en el Puerto Lomas, Arequipa, Perú.

\begin{tabular}{cccccc}
\hline Parásito & $\begin{array}{c}\text { Promedio } \\
\text { Abundancia } \\
\text { Media } \\
\text { Monoespecífica }\end{array}$ & $\begin{array}{c}\text { Promedio } \\
\text { Abundancia } \\
\text { Media } \\
\text { Recurrente }\end{array}$ & t & P \\
\hline Anisakis simplex & $1,65 \pm 0,88$ & $0,08 \pm 0,3$ & 16,85 & $<0.001$ \\
Anisakis physeteris & $3,69 \pm 2,49$ & $0,86 \pm 1,41$ & 8,42 & $<0.001$ \\
\hline
\end{tabular}

Tabla 6.- Infecciones monoespecíficas y recurrentes de dos

Ommastrephes angolensis Adam 1962, D. gigas e Illex coindetii (Verano 1837), quienes se comportan como hospederos intermediarios. De igual forma a $A$. physeteris se le ha encontrado en $O$. sagittatus y $O$. bartramii (Mattiucci \& Nascetti, 2006; Nigmatullin et al., 2009). Todos estos calamares se infectarían de Anisakis spp. al consumir macrocrustáceos planctónicos, peces micronectónicos y otros moluscos ommastréfidos (RosasLuis et al., 2008). Shukhgalter \& Nigmatullin (2001) han registrado $A$. physeteris en potas desde 90 a $431 \mathrm{~mm}$ y $A$. simplex solo en potas de mayor tamaño de 360 a $431 \mathrm{~mm}$. La mayor prevalencia de $A$. simplex con potas de mayor peso se relacionan con la variación ontogénica en relación a la alimentación de $D$. gigas. Los principales grupos en la dieta de D. gigas son los calamares Ommastrephidos, peces mictophidos y voladores (Exocoetidae) que se comportarían como hospederos paraténicos para $A$. simplex. Por lo tanto las potas de mayor peso por procesos de acumulación en Tabla 8. Variación entre años en el número de potas, LDM promedio y el tiempo de larvas de Anisakis al características de diversidad de los parásitos metazoos de las consumir estos items alimentarios infracomunidades de Dosidicus gigas entre 1981 y 2009.

\begin{tabular}{|c|c|c|c|c|}
\hline Descriptores & 1981-89 & 2003-2004 & 2008-2009 & 2009 \\
\hline Procedencia & $\begin{array}{c}\text { Aguas } \\
\text { oceánicas del } \\
\text { Pacífico-Este, } \\
\text { mayormente } \\
\text { de la Región } \\
\text { peruana }\end{array}$ & $\begin{array}{c}\text { Aguas } \\
\text { oceánicas del } \\
\text { Sur - Centro } \\
\text { de Chile }\end{array}$ & $\begin{array}{c}\text { Aguas } \\
\text { oceánicas } \\
\text { del Norte del } \\
\text { Perú }\end{array}$ & $\begin{array}{c}\text { Aguas } \\
\text { oceánicas del } \\
\text { Sur del Perú }\end{array}$ \\
\hline Número de potas & 849 & 124 & 42 & 254 \\
\hline LDM promedio (cm) & 23 & 61,1 & 69,8 & 60,2 \\
\hline Rango de LDM (cm) & $3-43$ & $30-92$ & $53,5-93$ & $39-88$ \\
\hline Riqueza de especies & 9 & 6 & 5 & 5 \\
\hline \multicolumn{5}{|l|}{$\begin{array}{l}\text { Índices de } \\
\text { diversidad }\end{array}$} \\
\hline Shannon-Wiener $\left(\mathrm{H}^{\prime}\right)$ & 0,67 & 0,32 & 0,12 & 0,41 \\
\hline Pielou (J') & 0,70 & 0,41 & 0,18 & 0,59 \\
\hline Simpson (S) & 0,27 & 0,58 & 0,81 & 0,43 \\
\hline Berger-Parker (d) & 0,45 & 0,73 & 0,89 & 0,54 \\
\hline
\end{tabular}

Larvas de tercer estadío de $A$. simplex han sido registradas a nivel mundial en varias especies de cefalópodos principalmente de la familia alimento clave (70\% de su dieta) de P. macrocephalus y la transmisión de $A$. physeteris desde $D$. gigas a este cetáceo marino (Rosas-Luis et al., 2008). incrementarían la prevalencia con el peso de $D$. gigas al ser similares entre potas de igual tamaño (Shukhgalter \& Nigmatullin, 2001). En el presente trabajo se encontró una alta prevalencia de ambas especies de Anisakis durante marzo (verano) en Arequipa, Perú. Shukhgalter \& Nigmatullin (2001) no encontraron diferencias cualitativas y cuantitativas en la prevalencia de infección en la fauna parasitaria de $D$. gigas para la región peruana en marzo-abril de 1981. Sin embargo, para la región EsteEcuatorial una mayor prevalencia de A. physeteris se observó en septiembre -1981 en comparación a febrero -1982. En adición existiría una estrecha relación entre $D$. gigas
Ommastrephidae: Todaropsis eblanae (Ball 1841), Ommastrephes sagittatus (Lamarck 1798), 
Tabla 9. Comparación entre potas parasitadas y no parasitadas con relación a la longitud dorsal del manto (cm) (LDM) en la Zona Sur del Perú, Puerto Lomas, Arequipa, Perú.

\begin{tabular}{|c|c|c|c|c|}
\hline & \multicolumn{2}{|c|}{ Promedio LDM (cm) } & \multirow[b]{2}{*}{$\mathrm{t}$} & \multirow[b]{2}{*}{$\mathrm{P}$} \\
\hline & $\begin{array}{c}\text { Potas } \\
\text { parasitadas }\end{array}$ & $\begin{array}{c}\text { Potas no } \\
\text { parasitadas }\end{array}$ & & \\
\hline Tentacularia coryphaenae & 60,97 & 60,97 & 1,77 & 0,07 \\
\hline Anisakis simplex & 61,23 & 60,09 & 0,90 & 0,36 \\
\hline Anisakis physeteris & 59,98 & 60,55 & 0,62 & 0,53 \\
\hline Total & 60,22 & 60,48 & 0,21 & 0,83 \\
\hline
\end{tabular}

Los tres estimadores no-paramétricos mostraron un valor 6 en relación al número de especies parásitas que debieran presentarse en el estudio, por lo tanto se infiere que se requiere más esfuerzo en el muestreo de D. gigas.

Los resultados obtenidos en el presente estudio evidencian un alto grado de sincronización parasíticotrófico en D. gigas (Shukhgalter \& Nigmatullin, 2001). Ambas especies de Anisakis de importancia zoonótica para el Perú, presentaron una prevalencia muy alta en $D$. gigas. El contagio se produce por el consumo de este molusco crudo en ceviche o insuficientemente cocinado. Por lo tanto se deben tomar medidas preventivas hasta el consumidor: 1) en producción primaria; 2) durante la captura y manipulación a bordo; 3) en la manipulación a tierra y 4) efectos térmicos durante la industrialización. De igual forma las entidades de Salud correspondiente deben tener programas de control de riesgos en establecimientos alimentarios, que incluyen la inspección, la elaboración y el almacenamiento de productos de pesca como la pota. Debe asegurarse, la inspección de comercios minoristas y de restaurantes. De igual manera, deberán implementarse Programas de Vigilancia y Control de contaminantes y residuos de alimentos. En muchos países la legislación aplicable incluye: 1) el garantizar un examen visual con el fin de detectar los parásitos visibles en la pota antes de ser puestos en el mercado mediante un plan de muestreo, 2) no poner en el mercado para uso humano productos de la pesca como la pota que estén claramente contaminados con parásitos, 3) congelación a una temperatura inferior a $-20^{\circ} \mathrm{C}$ por un mínimo de 24 h, y 4) la evisceración deberá llevarse de manera higiénica, esta medida es muy efectiva para Anisakidos que afectan a los peces. Sin embargo en el caso de la pota, aun eviscerada, el manto continúa presentando larvas de Anisakis (De la Torre et al., 2000; Cabrera \& Suárez-Ognio, 2002).

En 254 mantos de D. gigas en Puerto Lomas, Arequipa, Perú se encontraron formando parte de la comunidad de helmintos parásitos 5 taxas de helmintos endoparásitos: Didymozoidae gen. sp. no indet., $H$. trichiuri, T. coryphaenae, A. simplex y $A$. physeteris. T. coryphaenae es la especie de mayor prevalencia (56,29\%) y abundancia media (1,68\%) siendo ésta la de mayor cantidad encontrada en el músculo del manto. Los helmintos de importancia zoonótica están representados por A. physeteris (Prevalencia: 50\%) y A. simplex (Prevalencia: 15,74 \%). Se observó ausencia de relación entre la prevalencia de $A$. physeteris y A. simplex y la LDM del hospedero. Sólo se encontró relación lineal con la LDM de la pota y la prevalencia de $T$. coryphaenae. De igual forma la prevalencia de $A$. physeteris y $T$. coryphaenae no mostró relación lineal con el peso del manto del hospedero. Sólo la prevalencia de $A$. simplex mostró relación lineal con el peso estándar del manto. Se determinó que no existe relación entre la longitud dorsal del manto (LDM) y peso estándar del manto (PEM) de D. gigas y la abundancia de los parásitos encontrados. El promedio de LDM (cm) entre las potas parasitadas y no parasitadas con los tres helmintos parásitos es el mismo para T. coryphaenae, A. simplex y A. physeteris evaluados. Los valores obtenidos para las especies de helmintos zoonóticos $A$. simplex y $A$. physeteris muestran una disminución de la abundancia media cuando ambas especies se encuentran juntas. La prevalencia de las 2 especies zoonóticas $A$. simplex y A. physeteris durante el 2009 en Puerto Lomas fue mayor en comparación a 1981-1989, 2003-2004 y 2008-2009.

\section{Literatura citada.}

Alves D.R., Luque J.L. \& Paraguassú, A.R. 2002. Community ecology of the metazoan parasites of pink cusk-eel, Genypterus brasiliensis (Osteichthyes: Ophidiidae), from the coastal zone of the state of Rio de Janeiro, Brazil. Memórias do Instituto Oswaldo Cruz. 97: 683-689.

Benites C. \& Valdivieso V. 1986. Resultados de la pesca exploratoria de 1979/80 desembarque de cefalópodos pelágicos en el litoral peruano. Boletín Instituto del Mar del Perú. 1: 105-139.

Berland B. 1961. Nematodos from some Norwegian marine fishes. Sarsia. 2:1-50.

Bursey C.R., Goldberg S.R., \& Parmelee J.R. 2001. Gastrointestinal helminths of 51species of anurans from Reserva Cuzco Amazonico, Peru. Comparative Parasitology. 68:21-35.

Bush A.O., Lafferty K.D., Lotz J.L. \& Shostak A.W. 1997. Parasitology meets ecology on its own terms: Margolis et al. revisited. Journal of Parasitology. 83: 575-583.

Cabrera R. \& Suárez-Ognio L. 2002. Probable emergencia de anisakidosis por larvas de Anisakis physeteris durante el fenómeno El Niño 1997-98 en la costa peruana. Parasitología latinoamericana. 57: 166-170.

Cabrera R. \& Trillo A. P. 2004. Anisakidosis: ¿Una zoonosis parasitaria marina desconocida o emergente en el Perú? Revista Peruana de Gastroenterología. 24: 335342.

Campbell R.A. \& Callahan, C. 1998. Histopathological reactions of the blue shark, Prionace glauca, to post larvae of Hepatoxylon trichiuri (Cestoda: 
Trypanorhyncha: Hepatoxilidae) in relationship to scolex morphology. Folia Parasitologica. 45: 47-52.

De la Torre M.R., Pérez A.J., Hernández B.M., Jurado P.R., Martínez R.A. \& Morales F.E. 2000. Anisakiasis en pescados frescos comercializados en el norte de Córdova. Revista Española de Salud Pública. 75: 517526.

Dollfus R.P. 1942. Ètudes critiques sur les Tètrarhynques de Musèum de Paris. 19: 1-466.

Eiras J., Takemoto R \& Pavanelli G.C. 2000. Métodos de estudo e técnicas laboratoriais em parasitologia de peixes. Editora Universidade Estadual de Maringá, Maringá.

Esch G.W., Shostak, A.W., Marcogliese, D.J. \& Goater, T.M. 1990. Patterns and process in helminth parasite communities: an overview. p. 1-19. In: Esch G, Bush AC \& Aho J. (Eds.). Parasite Communities: Patterns and processes. New York. Chapman and Hall. 251p.

Gaevskaya A.V. 1977. Helminthofauna of the Atlantic squid Sthenoteuthis pteropus (Steenstrup). Science Reports of the High School Biology of Science. 8: 47-52.

Gaevskaya A.V. \& Shukhgalter O.A. 1992. Ontogenetic peculiarities of formation of the helminth fauna of squids of family Ommastrephidae. Ecology of Morya (Kiev). 40: 63-71.

Gaevskaya A.V., Nigmatullin Ch.M. \& Shukhgalter O.A. 1982. Data to study helminth fauna of squid Dosidicus gigas on the eastern Pacific. pp. 218-220. In: Problems of the Third All Union Conference, Kaliningrad, October 12-16, 1982. (Abstracts of Reports).

Gaevskaya A.V., Nigmatullin Ch.M. \& Shukhgalter O.A. 1983. Helminth fauna of three dominant species of squid of the family Ommastrephidae from southeastern Pacific and general remarks about its formation in this family. pp. 132-134. In: Starobogatov Y.I. \& Nesis K.N. (Eds.). Taxonomy and Ecology of Cephalopods. Zoological Institute of USSR Academy of Science, Leningrad.

Gaevskaya A.V., Nigmatullin Ch.M. \& Shukhgalter O.A. 1987. Geographical variability of ontogenetic dynamic of squid Dosidicus gigas helminth fauna in centraleastern Pacific. pp. 23-25. In: Parasitology and Pathology of Marine Organisms. Proceedings of the Fourth All Union Symposium, Kaliningrad, April 21-23, 1987.

Gómez P.R.M.C., Alvarez C.J.N. Segura P. L. \& Lamothe A.R. 1999. First record of Torticaecum sp. (Trematoda: Didymozoidae) in the chaetognath Serratosagitta serratodentata (Krohn 1853) from Caribbean waters. Journal of Plankton Research. 21: 1005-1008.

Gómez P.R.M.C., Álvarez C.J.N., Segura P.L., Lamothe A.R. 2007. Didymozoid Monilicaecum type trematodes in chaetognaths from Mexican Caribbean Sea. Revista Mexicana de Biodiversidad. 78: 483-487.

Hochberg F.G. 1990. Diseases of mollusca: Cephalopoda. pp. 47-227. In: Kinne O. (Ed.), Diseases of Marine Animals, Vol. III. Cephalopoda to Urochordata. Biologisches Anstalt Helgoland, Hamburg.

Iannacone J. \& Alvariño L. 2009. Catastro de la fauna endoparasitaria de la pota Dosidicus gigas (Cephalopoda) en el norte del Perú. Neotropical Helminthology. 3: 89-100.

Iannacone, J., Alvariño, L. \& Alayo, M. 2008. Aspectos ecológicos de los metazoos parásitos de Callichirus seilacheri (Bott 1955) (Decapoda, Callianassidae) en Lima, Perú. Neotropical Helminthology. 2: 9-17.

Iannacone J., Alvariño L., Guabloche A., Alayo M., Sánchez J., Arrascue A. \& Abanto M. 2003. Comunidades ectoparasitarias branquiales de la pintadilla Cheilodactylus variegatus Valenciennes 1833 (Pisces: Cheilodactylidae). Parasitología Latinoamericana. 58: 59-67.

Joyeux C. \& Baer J.G. 1936. Cestodes. Faune France. 30: 1613.

Justo M.C.N. \& Kohn A. 2009. Trematoda, Digenea, Didymozoidae, Coeliotrema thynni Yamaguti, 1938: First record in Brazil with three new hosts records. Check List. 5: 436-438.

Knoff M., De Sao Clemente S.G., Pinto R.M., Anfrede R.M. \& Gomes D.C. 2004. New records and expanded descriptions of Tentacularia coryphaenae and Hepatoxylon trichiuri homeacanth trypanorhyns (Eucestoda) from carcharhinids sharks from the state of Santa Catarina off-shore, Brazil. Revista Brasileira de Parasitologia Veterinaria. 13: 73-80.

Koyama T., Kobayashi A., Kumada M., Komiya Y., Oshima T., Kagei, N., Ishii, T. \& Mashida M. 1969. Morphological and taxonomical studies on Anisakidae larvae found in marine fishes and squids. Japanese Journal of Parasitology. 18: 466-487.

Maladineo I. 2006. Hepatoxylon trichiuri (Cestoda: Trypanorhyncha) plerocercoids in cage-reared northern bluefin tuna, Thunnus thynnus (Osteichthyes: Scombridae). Acta Adriatica. 47: 79-83.

Matsuura T., Sun S. \& Sugane K. 1992. The identity of Anisakis type II larvae with Anisakis physeteris confirmed by restriction fragment length polymorphism analysis of genomic DNA. Journal of Helminthology. 66: 33-37.

Mattiucci S. \& Nascetti G. 2006. Molecular systematic, phylogeny and ecology of anisakid nematodes of the genus Anisakis Dujardin, 1845: Un update. Parasite. 13: 99-113.

Moreno C. 2001. Métodos para medir la Biodiversidad. M\&T - Manuales y Tesis SEA. Cooperación Iberoamericana CYTED. UNESCO Orcyt. Sociedad Entomológica Aragonesa. $1^{\circ} \mathrm{Ed}$. México. 84 pp.

Nesis K.N. 1970a. Biology of the Peru-Chilean gigant squid, Dosidicus gigas. Okeanology. 10: 140-152.

Nesis K.N. 1970b. The biology of the giant squid of Peru and Chile, Dosidicus gigas. Oceanology of Washington. 10: 215-231.

Nigmatullin C.M., Nesis K.N. \& Arkhipkin A.I. 2001. Biology of the Jumbo Squid Dosidicus gigas. Fish Research. 54: 9-19.

Nigmatullin C.M, Shchetinnikov A.S \& Shukhgalter O.A. 2009. On feeding and helminth fauna of neon flying squid Ommastrephes bartramii (Lesueur, 1821) (Cephalophoda: Ommastrephidae) in the southeastern Pacific. Revista de Biología Marina y Oceanografía, 44: 227-235.

Overstreet R.M. \& Hochberg F.G. 1975. Digenetic trematodes in cephalops. Journal of the Marine Biological Association. 55: 893-910.

Palm H.W. 1995. Untersuchungen zur Systematic von Russelbandwwrmen (Cestoda: Trypanorhyncha) aus atlantischen Fishen. Beitr Institute Meeresk Kiel. 275 p. 
Palm H.W. 2004. The Trypanorhyncha Diesing, 1863. 710 p. PKSPL-IPB Press, Bogor.

Palm H.W. \& Walter T. 2000. Tentaculariid cestodes (Trypanorhyncha) from the Muséum national d'Histoire naturelle, Paris. Zoosystema. 22: 641-666.

Pardo-Gandarillas M.C., Duarte F., Chong F. \& Ibañez C.M. 2007. Dieta de tiburones juveniles Prionace glauca (Linnaeus 1758) (Carcharhiniformes: Carcharhinidae) en la zona litoral centro-sur de Chile. Revista de Biología Marina y Oceanografía. 42: 365-369.

Pardo-Gandarillas M.C., Lohrmann K.B., Valdivia A.L. \& Ibañez C.M. 2009. First record of parasites of Dosidicus gigas (D’ Orbigny 1835) (Cephalopoda: Ommastrephidae) from the Humboldt Current system off Chile. Revista de Biología Marina y Oceanografía. 44: 397-408.

Rodríguez O.B., García P.L. \& Pérez P.L.G. 2004. Checklist of helminth parasites of vertebrates in Costa Rica. Revista de Biología Tropical. 52: 313-354.

Roper C.F.E., Sweeney M. J. \& Nauen C.E. 1984. Cephalopods of the world. An annoted and illustrated catalogue of species of interest to fisheries. FAO:125277.

Rosas-Luis R., Salinas-Zavala C.A., Koch V. \& Del MonteLuna P. 2008. Importance of jumbo squid Dosidicus gigas (Orbigny, 1835) in the pelagic ecosystem of the central Gulf of California. Ecological Modelling. 218: 149-161.

Scholz T., Euzet L. \& Moravec F. 1998. Taxonomic status of Pelichnibothrium speciosum Monticelli 1889 (Cestoda: Tetraphyllidea) a mysterious parasite of Alepisaurus ferox Lowe (Teleostei: Alepisauridae) and Prionace glauca (L.) (Euselachii: Carcharinidae). Systematic of Parasitology. 41: 1-8.

Shukhgalter O.A. 1985. Oceanic squids of the southeastern Pacific as intermediate hosts in fish cestodes pp. 152153. In: Abstracts of Proceedings of the Seventh All Union Conference on Parasites and Diseases of Fish. Astrakhan.
Shukhgalter O.A. 1986. Squids of southeastern Pacific as reservoir hosts of tunas didimozoid trematodes pp. 166168. In: Vyalov, Yu.A. (Ed.)., Proceedings of the All Union Conference on Modern State of the Tuna Fishery and ecology of Scombroid Fishes, Kaliningrad, September 23-25, 1986.

Shukhgalter O.A. 1988a. The cestodes fauna of squids from South American oceanic waters pp. 89-90. In: Proccedings of the Third All Union Conference on Marine Biology, Sevastopol, October 18-20, 1988. Part II, Kiev.

Shukhgalter O.A. 1988b. Helminth fauna of squids from South American oceanic waters. Autoreferat dissertatsii na soiskanie uchenoj stepeni kandidata biologicheskikh nauk. Moscow. 23 p.

Shukhgalter O.A. 1992. The fauna of cestodes in squids from South American oceanic waters (southwestern Atlantic and southestern Pacific). Parasitologiya. 26: 130-140.

Shukhgalter O. A. \& Nigmatullin Ch. M. 2001. Parasitic helminthes of jumbo squid Dosidicus gigas (Cephalopoda: Ommastrephidae) in open waters of the central east Pacific. Fisheries Research. 54: 95-110.

Smith J.W. 1983. Anisakis simplex (Rudolphi, 1809, det. Krabbe, 1878) (Nematoda: Ascaridoidea): morphology and morphometry of larvae from euphausiids and fish, and a review of the life history and ecology. Journal of Helminthology. 576: 205-224.

Smith J.W. \& Wootten R. 1978. Anisakis and Anisakiasis. Advances in parasitology. 15: 93-163.

Stunkard H.W. 1977. Studies on tetraphyllidean and tetrarhynchidean metacestodes from squids taken on the New England coast. Biology Bulletin. Marine Biological Laboratory. (Woods Hole). 153: 387-412.

Yamaguti S. 1959. Systema Helminthum, Vol. II. The Cestodes of Vertebrates. Interscience, New York. 980 p.

Zuev G., Nigmatullin Ch. \& Nikolsky V. 1985. Nektonic Oceanic Squids (Genus: Sthenoteuthis). Agropromizdat Publishing House, Moscow. 224p.

\footnotetext{
${ }^{1}$ Museo de Historia Natural (MHN)-Universidad Ricardo Palma (URP). Av. Benavides 5440, Santiago de Surco, Lima, Perú.

${ }^{2}$ Laboratorio de Ecofisiología Animal, Facultad de Ciencias Naturales y Matemática, Universidad Nacional Federico Villarreal (UNFV), Av. Río Chepén s/n, El Agustino, Lima, Perú. Correo electrónico. joseiannacone@gmail.com

${ }^{3}$ Investigación y Desarrollo Tecnológico del Instituto Tecnológico Pesquero (ITP).
} 